\title{
WhiteCell: Energy-Efficient Use of Unlicensed Frequency Bands for Cellular Offloading
}

\author{
Pengfei Cui, Matthew Tonnemacher, Dinesh Rajan, and Joseph Camp \\ Department of Electrical Engineering, Southern Methodist University
}

\begin{abstract}
Cellular networks have addressed the multi-fold increase in traffic demand through various approaches from increasingly smaller cells to offloading demand to unlicensed spectrum, such as WiFi and TV white spaces. The latter approach has a tremendous cost benefit as unlicensed hardware can be colocated with existing cellular infrastructure. However, in these situations where demand is the greatest, WiFi spectral activity could be high and the total number of available white space channels are often inversely proportional to the population due to the existence of TV broadcast channels. Moreover, the additional hardware induces a higher energy demand of the cell site. In this work, we perform extensive spectral measurements of unlicensed bands in a major metropolitan area and crowdsource user mobility to consider potential swings in network load. Using these measurements, we design a queuing-based approach to serve these network demand dynamics in an energy-efficient manner according to differing qualities of service. In particular, we perform in-field experimentation of the diurnal spectrum activity across white space $(54-806 \mathrm{MHz})$ and WiFi (2.4 and $5.8 \mathrm{GHz}$ ) bands in typical settings across the Dallas-Fort Worth metroplex. We also consider mobility patterns from Androidbased crowdsourced measurements in four major Texas cities to infer the change in traffic demand induced by users. Driven by both data sets, we propose a Greedy Server-side Replacement (GSR) algorithm to estimate the power consumption for the use of unlicensed bands in cellular offloading. In doing so, we find that networks with white space bands reduce the power consumption by up to $513 \%$ in sparse rural areas over WiFi-only solutions via measurement-driven numerical simulation. In more dense areas, we find power consumption reduction across a 24 hour period to be, on average, $24 \%, 44 \%, 63 \%$ over WiFi-only offloading with one to three white space channels, respectively. Finally, we consider the quality of service impact on power consumption and find that up to $151 \%$ of the power can be saved with only a slight relaxation of waiting times.
\end{abstract}

\section{INTRODUCTION}

Cellular network providers have been forced to address multi-fold increases in traffic demand due to such factors as the ubiquity of mobile devices, the growth in on-the-go bandwidth needs such as streaming video and social media, and the rise of Internet-of-Things technologies. To meet this increasing demand, cellular carriers have shifted towards smaller cells and greater levels of offloading of user traffic to unlicensed frequency bands. In addition to the use of available $\mathrm{WiFi}$ channels, recent FCC changes have allowed the use of the white spaces of UHF bands formerly used exclusively for TV broadband services to now be used for data networks. These white space bands operate in available channels from 54-806 MHz, allowing far greater propagation range than WiFi bands for similar transmission powers [1]. However, data communication on the white space spectrum is only allowed when TV broadcast stations are not present, making the availability of white space channels inversely proportional to user population. Moreover, the co-location of unlicensed hardware with cellular hardware has implications on energy expenditure and ongoing network costs.

Unlicensed frequency bands have a wide range of propagation characteristics and policy differences (between 54$806 \mathrm{MHz}$ for white spaces and 2.4 and $5.8 \mathrm{GHz}$ for $\mathrm{WiFi}$ ). For the same transmission characteristics such as transmission power and antenna gain, the white space spectrum allows a propagation range of multiple times that of $\mathrm{WiFi}$ spectrum. The increased range of white spaces can be beneficial for low population densities (e.g., rural areas) since the user demand of a large area could be served by a single tower. However, for high population densities (e.g., dense urban areas) there are two issues that could make WiFi a more desirable option. First, the greater spatial reuse of WiFi can offer higher demand levels to be served. Second, in dense areas where user traffic demand is generally the highest, there are relatively few white space channels available. For example, there are no white space channels available in downtown New York City [2]. In most major cities in the United States, however, one to eight white space channels are available [2].

Unlicensed white space resources have previously been discussed as a WiFi-like resource [3]. In particular, solutions are actively being sought for the use of white spaces in data networks across the US and Europe [4], [5]. Most of these works focus on spectrum sensing and opportunistic access as opposed to cellular offloading. Previous cellular offloading works focus on the switching times and performance improvements for cellular, $\mathrm{WiFi}$, and other wireless technologies [6]. However, up to this point, the performance of white space spectrum in cellular offloading has not been fully investigated. Many works on mobile data offloading have focused on reducing the power consumption, and business models have been proposed [7]. For example, Han et al. [8] have shown an offloading strategy that reduces the power consumption through signal strength sensing among cellular and WiFi users. More general power saving strategies have also been explored [9]. Reducing powerer consumption saving not only benefits the carriers, but also reduces the cost for users. However, these works do not consider the large-scale channel variation and user mobility impacts on the capacity and power consumption of the offloaded network.

In this work, we perform measurements via spectral wardriving and crowdsourced user mobility to provide a measurement-driven characterization of residual channel capacity and total user demand, respectively, to gain a better 
understanding of these time- and spatial-dependent properties. We then design a queuing-based approach which considers the current channel capacity to serve the dynamic user demand in an energy-efficient manner according to differing qualities of service. Specifically, we perform extensive spectral measurements in the Dallas-Fort Worth metroplex at various times of the day to compose a diurnal pattern of spectral activity across $\mathrm{WiFi}$ and white space channels. Moreover, we examine mobility patterns from $60 \mathrm{k}$ users who are actively using our Android application and uploading signal strength measurements to a measurement repository. The measurementdriven spectral activity and load are used by our Greedy Server-side Replacement (GSR) algorithm which seeks to minimize the power consumption of the offloaded unlicensed network, considering the implications of varying qualities of service requirements.

In particular, our main contributions are as follows:

- We perform 24 hours of spectral measurements in diverse parts of the Dallas-Fort Worth metroplex, including neighborhoods, campus, a downtown business district, and an urban business district. Through these in-field measurements, we estimate the achieved channel capacity of these representative areas of a metropolitan region.

- We leverage our Android-based application, WiEye, to consider the mobility patterns of users in Dallas and three other large Texas cities: Houston, San Antonio, and Austin. Through these crowdsourced measurements, we anticipate the diurnal traffic demand dynamics of large metropolitan areas.

- Driven by these in-field and crowdsourced measurements, we formulate the wireless network structure as a queuing system, considering cellular, WiFi, and white space channels. We analyze the potential capacity and resulting energy consumption of the offloaded unlicensed network based on differing waiting times. Based on the analysis, we propose a Greedy Server-side Replacement (GSR) algorithm to allocate the unlicensed channel resources to minimize the power consumption.

- We perform measurement-driven numerical simulations to analyze various scenarios of unlicensed channel resources and users distributions. Our results show that the use of white space bands can reduce the power consumption in sparse areas by up to $512.6 \%$. We further show that the power savings can be up to $150.9 \%$ over a WiFionly configuration with the use of white space channels and relaxed but realistic waiting time constraints. Even in dense urban areas we show the average power consumption for a 24-hour period can be reduced by up to $63.3 \%$ over a WiFi-only network. Lastly, our analysis shows that while we achieve an average power consumption reduction of $19.6 \%$ over all the areas considered, we have analyzed a wide-range of realistic scenarios and established a framework to understand where the highest levels of gains will occur.

The rest of the paper is organized as follows. We describe the system and formulate the problem in Section II. Then, we present the queuing theory analysis and the Greedy Serverside Replacement (GSR) algorithm in Section II. We then

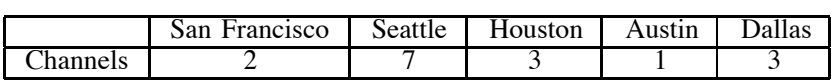

TABLE I

Number of White Space Channels in Major Cities

discuss our spectral analysis and crowdsourced measurements in Section III and resulting measurement-driven analysis of GSR in Section IV. We discuss related work in Section V and conclude in Section VI.

\section{WhiteCell FOR ENERgy-EFFiCIENT CELlulaR OFFLOADING}

\section{A. WhiteCell Network Architecture}

Wireless propagation refers to the signal loss characteristics when wireless signals are transmitted through the wireless medium. The strength of the received signal depends on both the line-of-sight path (or lack thereof) and multiple other paths that result from reflection, diffraction, and scattering from obstacles [10]. The widely-used Friis equation characterizes the received signal power $P_{r}$ in terms of transmit power $P_{t}$, transmitter gain $G_{t}$, receiver gain $G_{r}$, wavelength $\lambda$ of the carrier frequency, distance $R$ from transmitter to receiver, and path loss exponent $n$ according to [11]:

$$
P_{r}=P_{t}+G_{t}+G_{r}+10 n \log _{10}\left(\frac{\lambda}{4 \pi R}\right)
$$

Here, $n$ varies according to the aforementioned environmental factors with a value ranging from two to five in typical outdoor settings [12]. The propagation range of low-frequency white space channels is many times larger than high-frequency WiFi channels. For instance, $450 \mathrm{MHz}$ has more than 12 times the propagation range of $5 \mathrm{GHz}$ according to the Friis model. Thus, a single white space access point can cover an area up to hundred times of a WiFi access point (if the network was not capacity constrained). The large propagation of white space channels can potentially be applied for the reduction of network deployment costs [13], adaptation of vehicular dynamic access [14], or improvement of network capacity via additional channel resources [3]. However, previous works that focus on the application of many white space channel resources or point-to-point communication requiring a small amount of white space resources often fail to investigate the joint use of WiFi and white space bands with their respective advantages and disadvantages.

In wireless network design, a wider range of carrier frequencies allows greater flexibility and potentially better performance. White space frequency bands are a relatively new opportunity for wireless data networks which have yet to be fully deployed and exploited. However, the FCC restricts the number of white space channels in most dense urban areas to protect existing television broadcasts. The minimum number of white space channels in some major cities of the U.S are listed in Table I [2]. In New York City and Los Angeles (not listed), some districts have zero white space channel available. The densest urban areas of Austin have only one white space channel available. Most of the other cities in the table have 2 to 7 white space channels. Thus, insufficient channel capacity exists to serve all urban users in the coverage region of these cities with a strictly white space network. Moreover, 


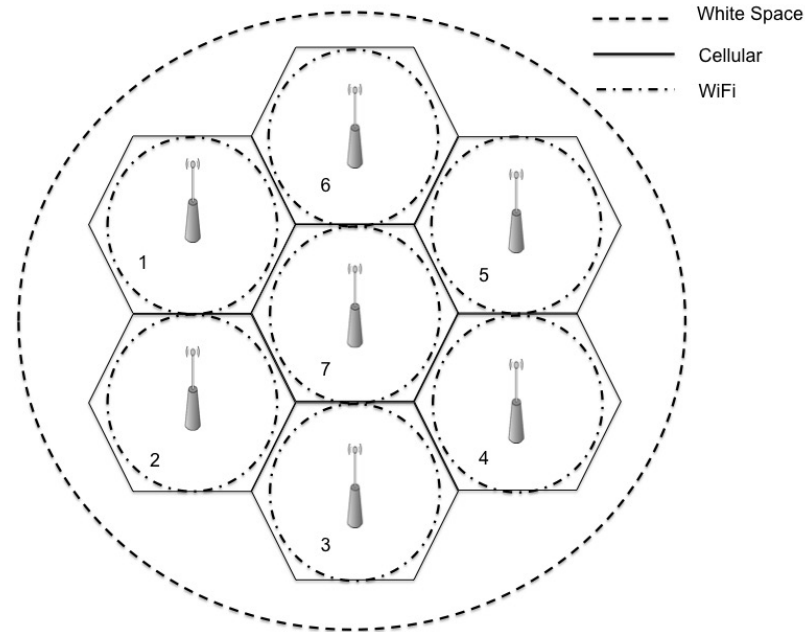

Fig. 1. WhiteCell Structure

most of the dense urban areas already have deployed cellular (and potentially WiFi) networks, which should be exploited jointly with white space networks. Our proposed wireless system leverages the existing infrastructure, adding additional frequency efficiency and lower energy usage.

Here, we introduce a wireless network structure that leverages existing cell sites but additionally uses $\mathrm{WiFi}$ and white space radios for offloading of mobile traffic. We assume that each cell contains a cellular and WiFi transmitter. However, due to the range of white space transmitters, we assume that the central cell can cover all six surrounding adjacent cells with at least one white space transmitter (as shown in Fig. 1), forming a WhiteCell. We assume that these seven smaller cells that compose one WhiteCell can operate independently of surrounding WhiteCells due to spatial reuse. For our analysis, we will consider up to three white space transmitters and channels are available per WhiteCell, subject to the availability of white space channels in that geographical region. The system can adapt the channel resource allocation to reduce power consumption according to the variation in traffic demand. The $N$ users that exist in the WhiteCell network architecture have $M$ access points equipped with $\mathrm{WiFi}$ and cellular capabilities and $M / 7$ cells equipped white space capabilities. $F_{w}$ is the number of white space radios installed on the central cell's access point. We assume each radio has a channel capacity of $C$ if the channel is free of spectral activity (i.e., if the channel is idle) and reduced according to the percentage of time the channel is in use. We assume that sufficient memory space exists to buffer traffic to the users from each access point. For this work, we consider only the offloaded traffic to the unlicensed bands and the power consumption thereof. Hence, the traffic aggregated at each access point could be served via WiFi in each smaller cell or via white space in the larger WhiteCell. The traffic is served in a first-in-first-out (FIFO) scheduling strategy. In this structure, the traffic of each cell could be transferred by $F_{w}+F_{m}$ channels. Here, $F_{m}$ is the number of WiFi channels used in addition to the $F_{w}$ white space channels. We assume the users in the same cell are in a single interference domain.

Instead of assuming the wireless channels are on-off [15] or have equal capacity, we apply a measurement-driven estimation to get the achieved channel capacity. The capacity of the channel between the access points and each user is noted as a matrix in the following way:

$$
H_{i, j}^{f}(t)=G(\zeta, t), i \in M, j \in N, f \in\left(F_{M}+F_{w}\right)
$$

Here, $\zeta$ represents the in-field measured historical data and dynamic sensing information. A context-aware method is applied to estimate the $j$ th user capacity $H_{i, j}^{f}(t)$ to an access point $i$ on channel $f$. The users in a single cell have the same channel status. We assume the channel capacity is flat during a certain time slot. The switching time is negligible in the system. The calculation of achieved channel capacity is introduced in Section IV-A. The traffic demand of a user obeys a Poisson process, with the vector noted as $D(t)=$ $\left[D_{1}(t), D_{2}(t), \ldots D_{N}(t)\right]$ and the sum rate $D(t)=\sum_{i=1}^{N} D_{i}(t)$ The rate $D(t)$ is the aggregate rate of data generated from all users.

During a time slot, the unscheduled radios remain in sleep mode to save energy. We also ignore the sleeping energy as well as the amount of energy spent on channel or radio switching. An operating radio will cost equal standby power per unit time. Previous human factors research [16] shows that users have a certain level of patience for a response. The tolerance time of users varies across the traffic type, such as text information, voice and video. To simplify the problem, we assume an average value for tolerance response time $W$ of all the users in the system. The channel capacity is the maximum achievable per spectral resource. A wireless system could serve users faster with less response time via more channel capacity. While at the same time, the power consumption increase with the amount of spectral resources allocated. Thus, if users have a less strict requirement on the response time, less channel resources (bandwidth and energy) are necessary.

\section{B. Problem Formulation and System Model}

We formulate the wireless network system introduced in Section II-A as a discrete-time queuing system shown in Fig. 2. The channels are represented as servers in the queuing model. Table II summarizes the notation used in this work. In the system, there are $F_{w}$ white space radios and $M$ cells. $F_{M}$ represents the WiFi channels allocated to each of the $M$ cells. Multiple cells may share the same WiFi channel, but we assume there is no overlap of their service areas due to spatial reuse. Thus, the queuing system has $M$ queues of the cells and $F_{M}+F_{w}$ servers in total, which are connected via timevarying $\mathrm{WiFi}$ and white space channels $H^{*}\left(M, F_{M}+F_{w}\right)$.

The matrix $\left\{S_{i, j}(t), i \in\left(F_{M}+F_{w}\right), j \in M\right\}$ denotes the wireless resource association as shown below:

$$
S_{i, j}(t)= \begin{cases}1 & \text { if } D_{j \in M}, \text { is associated with } \\ & \text { radio } i \in\left(F_{M}+F_{w}\right) \\ 0 \quad \text { Otherwise }\end{cases}
$$

To maintain the quality of service, the queuing system restricts the expected response time of the system $w$ to no more than the tolerance threshold $W$ :

$$
E[w] \leq W
$$




\begin{tabular}{ll}
\hline$t$ & Time slot \\
$N$ & Set of users \\
$M$ & Set of WiFi cells \\
$H_{i j}^{f}$ & Measurement based Capacity between AP i and user j on channel f \\
$F_{m}$ & WiFi Channels in the cells \\
$F_{w}$ & Set of White Space Channels \\
$S(t)$ & User access channel schedule \\
$A$ & Activity Level \\
$C$ & Clean Radio Capacity \\
$D$ & User Demand \\
$R$ & Operating Radio \\
$\zeta$ & In-Field Measurements \\
$W$ & User Tolerance time window \\
$\mu$ & Channel capacity assigned for a cell \\
$P_{s}$ & Standby Power Consumption \\
$P_{t}$ & Transmission Power Consumption \\
\hline
\end{tabular}

TABLE II

TABLE OF NOTATIONS

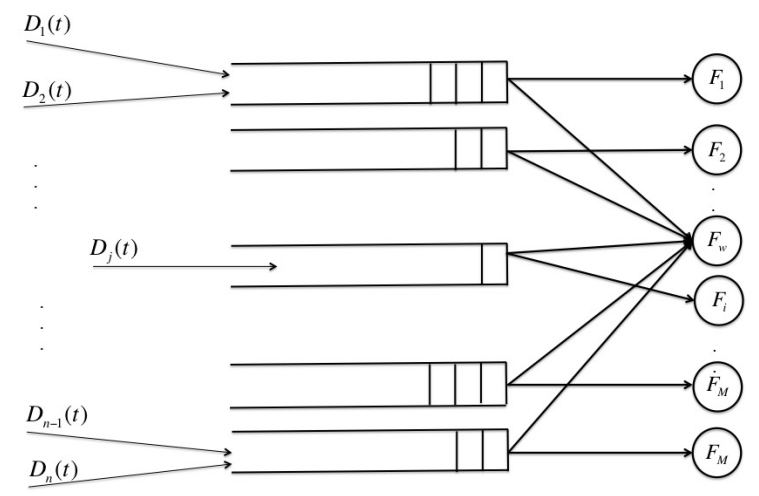

Fig. 2. System Model

When the total traffic demand of the users in the system is relatively small, the channel capacity of a single white space channel could achieve the quality of service in response time for all the users. In this scenario, all cell radios could be switched to sleep mode for power saving. On the other hand, as the traffic demand increase with the number of users or the demand per user, the channel resources need to be increased as the number of servers in the queuing system increases to meet the user response time tolerance requirements. Thus, in this extreme case, all the radios have to keep operating to provide the appropriate quality of service. Moreover, when the users are distributed non-uniformly, the white space channels are able to deliver more capacity for the cells with more traffic to balance the system load without adding new infrastructure. The flexibility of white space channels offers new opportunity for power saving and offered load adaptation in network design.

In this work, we focus on analyzing the power savings for the WhiteCell system. To model the power consumption of the system, we sum the power consumption of each operating radio in two power consumption categories: standby and transmission. We assume the sleeping or standby power consumption is negligible as well as the on-off switching power consumption. We define $R_{i}$ as radio status of each of the $i \in F_{w}, F_{M}$ radios in the system. The value of $R_{i}$ denotes the power consumption of the radio for standby and transmission. When the radio is in sleeping mode, $R_{i}=0$. Otherwise, the
$R_{i}$ is defined as follows:

$$
R_{i}(t)= \begin{cases}P_{s}+P_{t} \cdot \mu & \sum_{j=1}^{N} S_{i, j}(t) \geq 1 \\ 0 & \text { Otherwise }\end{cases}
$$

Here, $P_{s}$ is the standby power consumption of a radio, which is a constant value, $P_{t}$ is the unit transmit power consumption of the assigned channel capacity, and $\mu$ is the allocated channel capacity of the radio. $R_{i}(t)$ is the power consumption of the radio during the time slot.

Thus, to reduce the power consumption of the system, it could be implemented via minimizing the sum of $R_{i}$ under the quality of service constraints. The objective is to minimize the power consumption of the system:

$$
R^{*}(t)=\min \left\{\sum_{i=1}^{\left(F_{M}+F_{w}\right)} R_{i}(t)\right\}
$$

where $R^{*}$ represents the minimum operating power consumption required of the system. The allocated resources $\mu$ could be adjusted to approach the minimum power consumption in the model. According to the queue-based quality of service model and power consumption model, we further analyze the WhiteCell system and propose our greedy solution.

\section{WhiteCell Challenges And Analysis}

Prior works formulate similar multi-channel systems as $M / M / m$ queuing systems for analysis [15]. However, the WhiteCell system is not able to be formulated as such due to the non-equal assignment of channel capacity to each cell across white space and WiFi channels. Thus we first analyze the queuing system and apply the previous work on $M / M / m$ queuing theory to approach a solution for the system.

In the WhiteCell system, the users of a cell have access to both the WiFi channel assigned for the cell and the white space channels, which covers one WhiteCell or seven adjacent $\mathrm{WiFi} /$ cellular cells. The division of white space channel capacities forces capacity variation of the servers in the queuing model, thereby removing the equal service capacity assumption of the queuing system. However, the equal server capacity is a requirement for a general $M / M / m$ queuing system analysis. Thus, the $M / M / m$ queuing system of a multi-channel version is not directly applicable for this WhiteCell system model.

The response time $W$ represents the duration of time from when user sends a request to when the server responds. In the system design, the response time $W$ constraint has to be satisfied to maintain the quality of service. The channel quality in multiple cells varies throughout a single time slot, which is mentioned as part of multi-user diversity in previous works. Multi-user diversity is a form of diversity inherent in a wireless network provided by independent time-varying channels across the different users [17]. The diversity could be generated by the interference from a device inside or outside of the network as well as environmental variations. Some cells may have idle white space channels while the other cells may suffer from TV-broadcast occupancy or other spectral activity. To address the variation, we utilize in-field measurements to 
infer channel capacity in this work as opposed to an on-off channel availability assumption. We define the activity level as the percentage of time that the measured signal strength exceeds a threshold to estimate the residual capacity of the channel. We perform measurements to sense the spectral activity in multiple locations in North Texas through our portable spectrum analyzer (discussed in more detail in Section III-A). The percentage of sensing samples $\left(\delta_{\theta}\right)$ above an interference threshold $(\theta)$ over the total samples $(\delta)$ per unit of time is the activity level $(A)$ :

$$
A=\frac{\delta_{\theta}}{\delta_{a}}
$$

The capacity of a clean channel is denoted by $C$. With the protocol model, the achieved capacity of a channel $C_{r}$ could be represented as the remaining free time of the channel capacity according to:

$$
C_{r}=C *(1-\bar{A})
$$

$\bar{A}$ represents a long term activity level measurement.

The user mobility footprint is one of the key points in the wireless resource allocation. As more users stay in or move into a cell, the more wireless channel resources are need to be allocated to the cell to serve the demand. Thus, the user distribution is a critical aspect of resource allocation. The large propagation of white space channels allows them to be used flexibly over a WhiteCell to balance the non-uniform user distribution by compensating channel allocation for additional capacity on demand. To identify the user mobility patterns, we analyze the data set from WiEye, an Android application that reports the location, velocity, and signal information to leverage the mobility patterns of users. The user mobility footprints are derived from leveraged from crowdsourcing thousands of users. The setup and results are shown in Section IV.

With these measurements, we further analyze the channel capacity allocation for the WhiteCell system. We first investigate the channel capacity allocation sub problem in a single cell $(1 / 7 t h$ of a WhiteCell). The users of the the same WiFi cell are in a heterogeneous queuing system with servers of WiFi channels and white space channels with each having a capacity represented by $\mu_{1}, \mu_{2}, \ldots \mu_{\left(F_{w}+F_{m}\right)}$. Here, $\mu$ denotes the capacity of a channel allocated in this cell for all channels. There are multiple accessible channels in the cell: the WiFi channel and the white space channels, which could be allocated to other small cells within the same WhiteCell. Since the white space channel is divided over seven cells and that cell has at least one dedicated WiFi channel, the capacity of a white space channel division is typically the smallest portion of channel capacity in a cell. Therefore, there are three cases of channel capacity comparisons in a single cell.

The first case occurs when two or more channels are assigned to the cell and the capacity of the least contributing white space channel is several times less than the maximum channel capacity (typically a WiFi channel). In Eq. 12, we see that this occurs when the maximum over minimum ratio is greater or equal to 2. An example of this scenario could be when one WiFi channel and one white space channel exist and the white space capacity is equally divided $(1 / 7 t h)$ across the cells that compose a WhiteCell.
For the first case, called a heterogeneous server queuing system, we apply the transformation model in [18] to estimate the response time $\bar{w}$. In the transformation model, the actual arrival rate for one specific server $\lambda_{s}$ is defined as:

$$
\lambda_{s}=D_{\text {cell }} /\left(F_{c W}+F_{c M}\right)
$$

where $D_{\text {cell }}$ is the traffic aggregated from the users in the cell, $F_{c W}$ represents the set of white space channels assigned in the cell, and $F_{c M}$ notes the WiFi channels in the cell. $\lambda_{s}$ in terms of statistic average for a specific channel in this work.

The other parameters are defined in Eq. 10 through 12.

$$
\begin{gathered}
\mu_{\text {min }}=\min \left(\mu_{1}, \mu_{2}, \ldots \mu_{\left(F_{w}+1\right)}\right)=\bar{\mu} \\
\mu_{\max }=\max \left(\mu_{1}, \mu_{2}, \ldots \mu_{\left(F_{w}+1\right)}\right) \\
k=\left\lfloor\frac{\mu_{\max }}{\mu_{\min }}\right\rfloor
\end{gathered}
$$

When $k \geq 2$ the average response time of such a heterogeneous system [18] could be represented as in Eq. 13:

$$
\bar{w}=\frac{1}{\frac{1}{3} \bar{\mu}(2 k+1)-\lambda_{s}}
$$

Through the transformation model, we can further search the channel capacity required for the response time constraints. Additionally, the power consumption can be found for the system.

The second case is one in which only a single channel serves the cell, either the $\mathrm{WiFi}$ channel or part of a single white space channel. This case can be simplified to a $M / M / 1$ queuing system that only has one server in the model. When the traffic is able to be served by part of a single white space channel or the WiFi channel, as in the second scenario, the system converges to a $M / M / 1$ queue. The response time $\bar{w}$ can then be estimated from Eq. 14 [19].

$$
\bar{w}=\frac{1}{\mu^{+}-D}
$$

$\mu^{+}$represents the channel capacity of the single channel capacity allocated in the cell.

The third case (like case one) also has more than one channel operating in the cell. However, the key distinction from case one is that the capacity of the channels are approximately the same (i.e. when $k=1$ ). This system becomes a queuing system, which has multiple equal capacity servers in the model. We remark it as a homogeneous $M / M / m$ system, and the first case as a heterogeneous $M / M / m$ system.

The average response time can be found through a search strategy [19]:

$$
\bar{w}=\frac{1}{\mu^{*}}\left(1+\frac{c(m, \rho)}{m(1-\rho)}\right) \approx \frac{1}{\mu^{*}} \frac{1}{1-\rho^{m}}
$$

where $\mu^{*}$ is the average capacity of channels in the $M / M / m$ queuing system. A half-search strategy is applied to find the minimum value of $\mu^{*}$ to reduce the power consumed by transmission. Here, $\rho=\frac{\lambda}{m \mu^{*}}$ is the traffic density, and $c(m, \rho)$ is the Erlang-C formula [19]. 


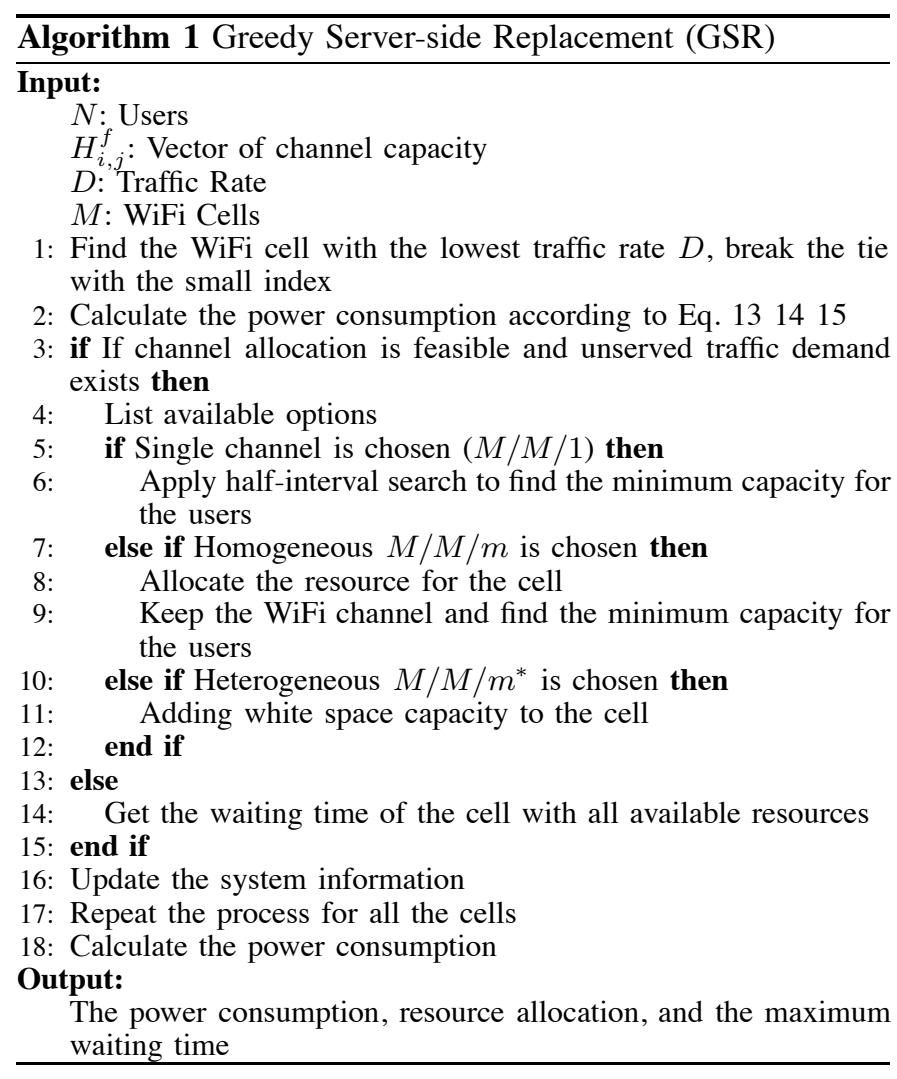

In the WhiteCell, the less radios that are in operation, the less power is consumed by the system as shown in Eq. 5. The central idea of power saving in each WhiteCell is to alleviate the use of the decentralized WiFi radios with the centralized white space channels. To implement the division of the white space capacity, we propose a Greedy Server-side Replacement (GSR) algorithm to minimize power consumption in the system as shown in Algorithm 1.

The GSR algorithm inputs are the measurement-based residual channel capacity, the crowdsourced user distribution information, the number of white space channels, and the WiFi cells. The more cells that can be served by a largelypropagated white space channel, the less radios need to be turned on. To turn off more radios, the algorithm starts to replace the cell radios with white space channels in the cells with less traffic demands. The algorithm compares the three configurations of channel capacity assignment in each cell and chooses the setup with the lowest power consumption. Further, the process is repeated until all traffic demand is served or the channel resources have been used up. Finally, GSR outputs the power consumption and channel allocation of the system.

\section{IN-FIELD EXPERIMENTATION}

In this section, we introduce our in-field measurement experimentation, consisting of both spectral wardriving and crowdsourcing of mobility patterns using an Android application. In doing so, we compose an empirically-driven understanding of diurnal spectral activity in diverse areas of representative metropolitan areas and infer diurnal shifts in user demand as users move throughout a region.

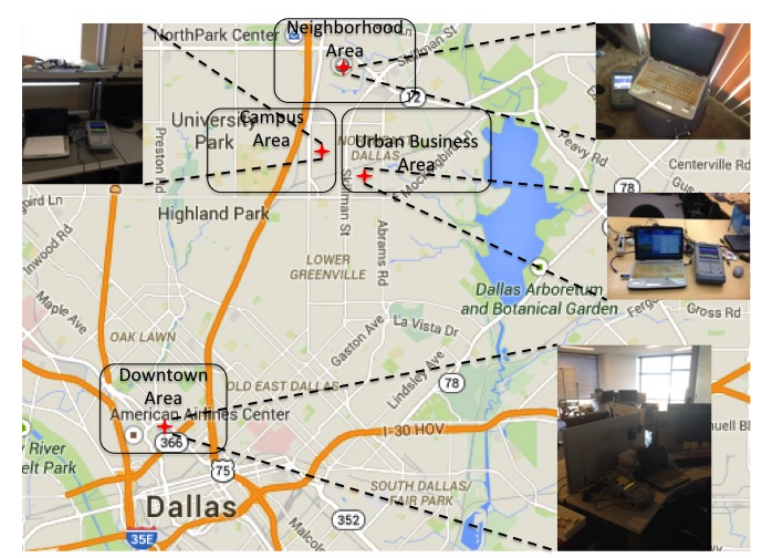

Fig. 4. Long Term Measurements Locations

\section{A. Spectral Wardriving}

When considering networks that can leverage a wide range of carrier frequencies, an in-situ understanding of spectral activity is critical in determining the optimal network configuration. To this end, we collected spectral user activity measurements in several typical areas including urban and suburban business districts and neighborhoods and a university campus in Dallas, TX, as seen in Fig. 4.

In each of the locations, we collect channel state information measurements for a 24-hour period during weekdays. For spectral analysis, we employ a Rohde \& Schwarz FSH8 portable spectrum analyzer that operates in frequencies from $100 \mathrm{KHz}$ to $8 \mathrm{GHz}$. This portable spectrum analyzer is controlled by a Python script on a laptop that records the received signal strength over time. To the best of our knowledge, there is no commercially-available, mobile, multi-band antenna from 450 $\mathrm{MHz}$ to $5.2 \mathrm{GHz}$ on the market. Due to this limitation, we use a $700-\mathrm{MHz}$ mobile antenna to perform in-field measurements and normalize the mobile antenna gain across all bands with indoor experimentation. To do so, we use a Universal Software Radio Peripheral (USRP) N210 to generate signals at 450 $\mathrm{MHz}, 800 \mathrm{MHz}$, and $2.4 \mathrm{GHz}$. We feed these signals directly to the spectrum analyzer and adjust the configuration of USRP to make the received signal strength the same as the 5.2 $\mathrm{GHz}$ signal from Gateworks 2358 with a XR5 radio. We then connect the signal source to a fixed multiband antenna (QT 400 Quad Ridge Horn Antenna) and measure the received signal at a fixed distance with the $700 \mathrm{MHz}$ antenna and antennas for different bands to obtain the antenna loss for each band. We adjust the in-field received signal strength collected via the 700-MHz mobile antenna according to this normalization. After the adjustment, any received signal over $-79 \mathrm{dBm}$ is denoted as activity on the channel.

When wireless devices operate in WiFi bands, the channel separation and resulting differences in propagation are relatively small (e.g., $5 \mathrm{MHz}$ for the $2.4 \mathrm{GHz}$ band). As a result, many works assume that the propagation characteristics across different channels are similar. However, with the large carrier frequency differences of $\mathrm{WiFi}$ and white space bands (e.g., multiple $\mathrm{GHz}$ ), propagation becomes a key factor in the deployment of wireless networks with both bands. Here, a frequency band is defined as a group of channels which have little frequency separation, meaning they have similar 


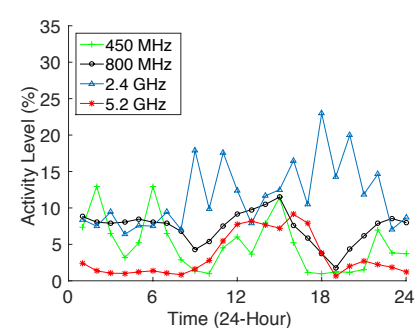

(a) Urban

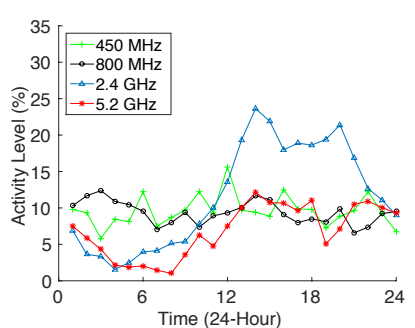

(b) Downtown

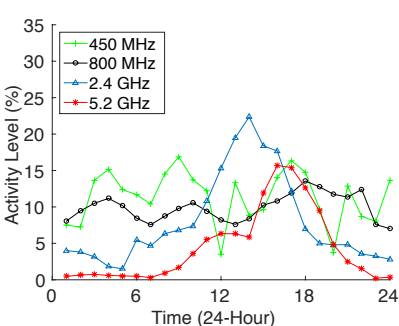

(c) Campus

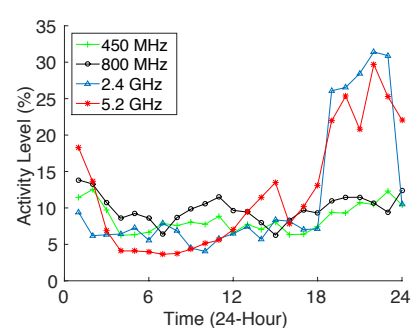

(d) Neighborhood

Fig. 3. Measured Activity Level (Percentage)

propagation characteristics. In this work, we consider the diverse propagation characteristics for four frequency bands: $450 \mathrm{MHz}, 800 \mathrm{MHz}, 2.4 \mathrm{GHz}$, and $5.2 \mathrm{GHz}$. We refer to the two former frequency bands as white space bands and the two latter frequency bands as WiFi bands. The differences in propagation and spectrum utilization create opportunities for their joint use in cellular offloading according to the environmental characteristics (e.g., urban or rural and downtown or residential) of the deployment location.

Through the measured data set, we are able to calculate the activity level via Eq. 7 with respect to the $-79 \mathrm{dBm}$ threshold. We calculate a single activity level per one-minute time window, and then average these activity levels over each hour across the 24-hour duration as shown in Fig. 3.

Through the activity-level results in Fig. 3, we can see several interesting differences between frequency bands, time, and locations. The frequency of variation of $450 \mathrm{MHz}$ and $800 \mathrm{MHz}$ is relatively large compared to the bands $2.4 \mathrm{GHz}$ and $5.2 \mathrm{GHz}$ which have more of a daily pattern. In fact, the $450 \mathrm{MHz}$ band has more variation in the downtown area than other areas, whereas the $800 \mathrm{MHz}$ band has similar behavior across the four areas. The $800 \mathrm{MHz}$ band is used for emergency communication, which may explain the dynamics of the spectral activity in all areas across time. We also observe that the maximum activity levels of the 2.4 and $5.2 \mathrm{GHz}$ bands occur in the neighborhood, which could be explained by home WiFi that are increasingly dual band. In the urban area, the activity of $2.4 \mathrm{GHz}$ has two peaks in most activity around 10:00 AM and 4:00 PM. In the downtown area, the single peak of $2.4 \mathrm{GHz}$ spans from 11:00 AM to 8:00 PM. In the campus environment, the peak starts at 11:00 AM which is a popular time for courses being taught in the building. The spectral activity peak for the neighborhood area with $2.4 \mathrm{GHz}$ starts at 6:00 PM and lasts until 11:00 PM. The 5.2 GHz band has a similar peak but lower activity than $2.4 \mathrm{GHz}$ and with a peak in neighborhood at around 9:00 PM. We integrate these activity level results into the analysis of our GSR algorithm in the following section.

\section{B. Crowdsourcing User Mobility}

To identify user mobility patterns, we leverage the data from our Android-based application, WiEye, to analyze user locations and mobility across time. The WiEye application, created for the data collection, is currently available for download and use via the Google Play store. Our application offers connection quality information for WiFi access points in both graphical and tabular form as a download incentive.
There are over $60 \mathrm{k}$ active users throughout the world, which have collected a total of over 200 million measurements of WiFi and cellular signal strengths. All data collection is done as a background process: for opted-in users, signal strength measurements are performed periodically as a uniform random interval from 4 to 6 hours or for SMU researchers, who continuously log signal strength measurements to make the most of the time in the field. Additionally, the data collected has been approved by the Southern Methodist University Institutional Review Board, a human subjects research committee, ensuring that all ethical precautions have been taken in collecting user data with our application.

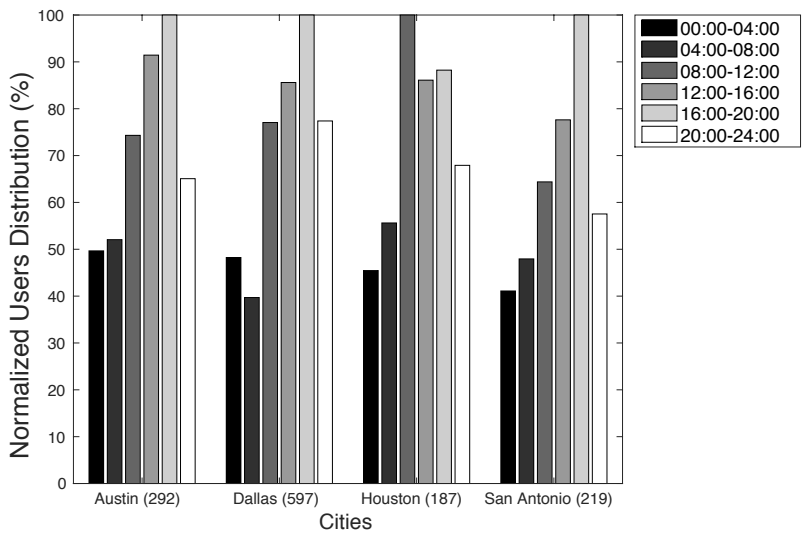

Fig. 5. Total User Variation Across 4-hour Time Slot

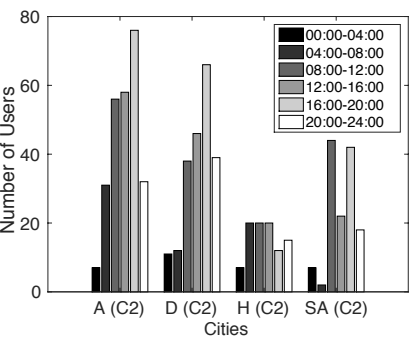

(a) Max Fluctuation Tile

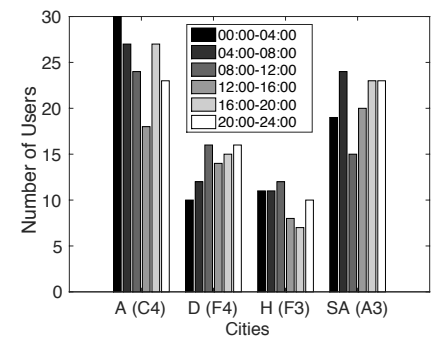

(b) Min Fluctuation Tile
Fig. 6. User Distribution, A: Austin, D: Dallas, H: Houston, SA: San Antonio

User mobility data has a direct impact on the power consumption and resource allocation of a wireless network. For our user mobility analysis, we pull data from the WiEye database in several Texas cities, including Houston, Dallas, San Antonio, and Austin in weekdays from October 1st 2014 to March 20th 2015. Since we assume large downloads do not occur at high mobility, we removed all data points 


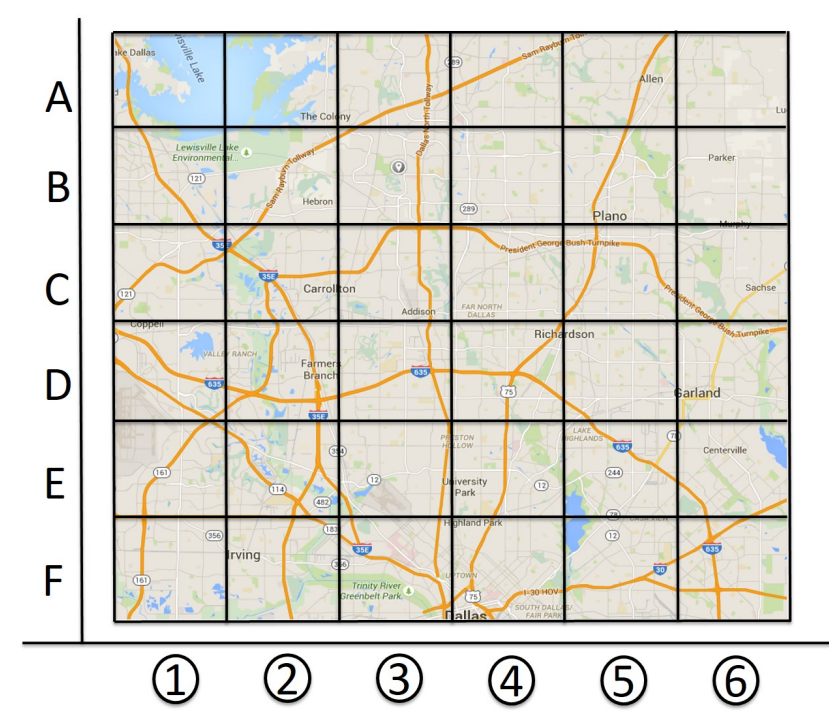

(a) Dallas

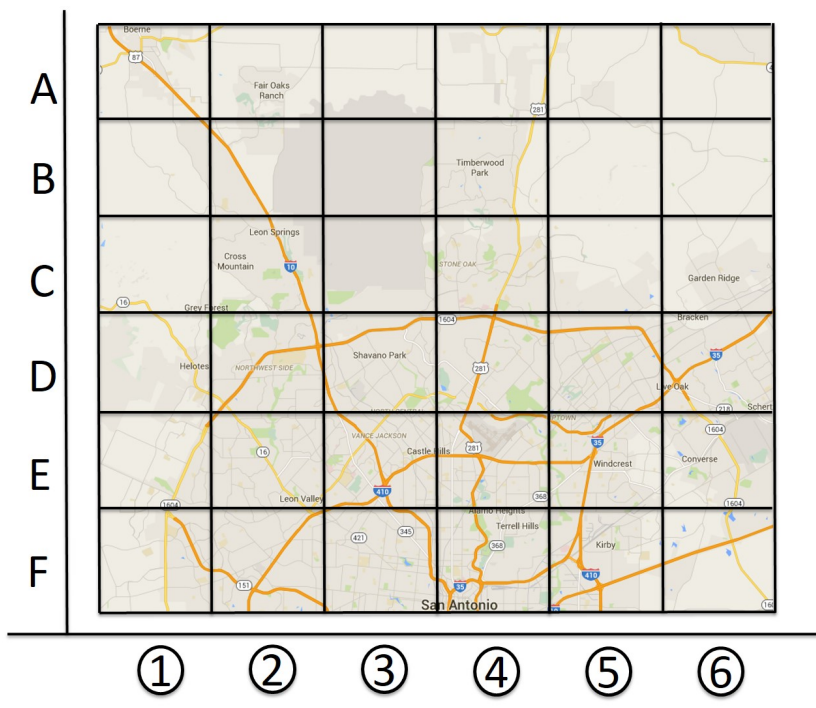

(c) San Antonio

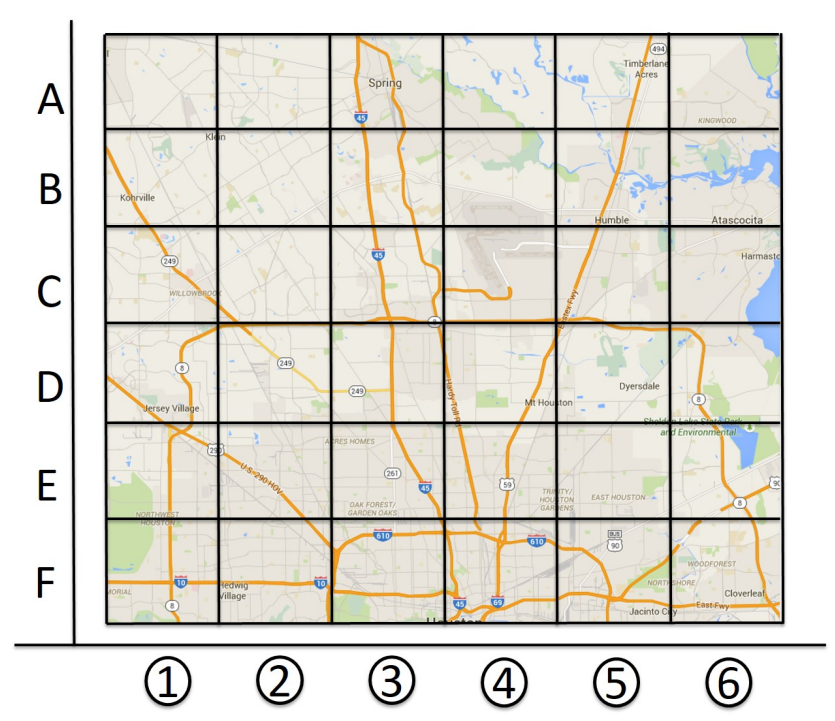

(b) Houston

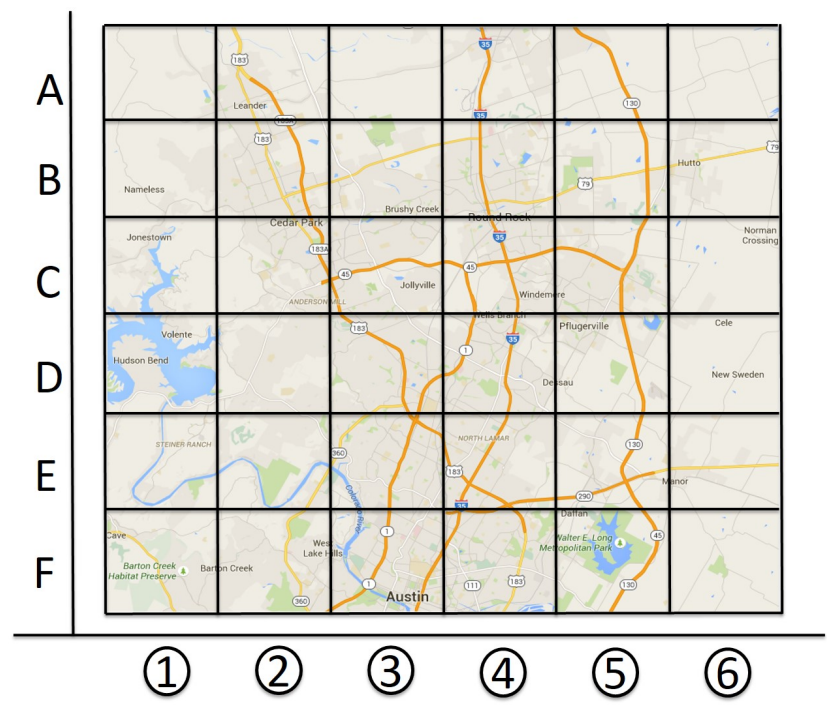

(d) Austin

Fig. 7. WiEye Regional Grids

with reported speeds above $30 \mathrm{~km} / \mathrm{h}$. We first query the all Dallas measurements in the range restricted from $32.7471 \mathrm{~S}$ to $33.2153 \mathrm{~S}$ latitude, and from $97.1677 \mathrm{~W}$ to $96.5600 \mathrm{~W}$ longitude. We then superimpose the same area from Dallas over Houston, Austin, and San Antonio according to the difference of latitude and longitude between the center of the cities as defined by Google maps. We split each area into a 36-region grid as shown in Fig 7, quantize time into six 4hour slots per day, and record in each grid the number of unique users according to the device ID records from the WiEye database. We compress the data set into one weekday since WiEye reports the measurements only periodically, and the data set has a limited number of users for a given region.

The total users are shown in Fig. 5, but due to space limitations, the detailed results of each tile are available upon request. From the data set, we identify up to 597, 292,
219, 187 users in Dallas, Austin, San Antonio and Houston, respectively. There are several interesting findings in user numbers that we can take away from the data set. The number of user peaks happen at the time slot between 16:00-20:00 in Dallas, Austin, and San Antonio, the exception being Houston during the 8:00-12:00 slot. The lowest number of users occur at the time slot between 0:00-4:00 in Houston, Austin, San Antonio while between 4:00-8:00 in Dallas.

In Fig. 6(a), the tile that has the most fluctuation in Dallas is C2 with a $600 \%$ swing and is a residential area with multiple highway intersections. In Fig. 6(b), F4 has the least fluctuation with a $60 \%$ swing and includes part of a downtown and dense urban area of Dallas. The other 3 cities also have C2 tile with the most user fluctuation, Austin (1100\%), Houston (285.7\%), and San Antonio (1466.7\%). The least fluctuating tiles are C4 in Austin with $66.7 \%$ variation, F3 in Houston with $71.4 \%$ 
variation, and A3 in San Antonio with 46.7\% variation. The number of users in tile F4 of Dallas fluctuate less than other tiles. A similar phenomenon is seen in F4 of the Houston measurements. Since we have few data points collected from the area in Austin and San Antonio, it is not clear of the fluctuation in downtown area of these two cities. Grid regions A2 and B2 of Dallas both contain part of a lake, but B2 always has more users than A2, most likely caused by the highway in grid B2. Similar highway influences can be seen in D5, C5. Regions C3 and D3 have more users than other regions in column 3 across almost nearly all time since they have highway intersections. In Austin, we find that more than $70 \%$ of users are located in 3 tiles: C2, C3, and C4. There are several companies located in these tiles, such as Samsung semiconductor, which seem to attract most of our users in Austin. With the limited number of measurements, it is hard to discern all of the factors that lead to user mobility. Since we have the highest density of measurements in Dallas, our analysis is based on Dallas for the rest of the paper. In the future, more patterns are able to be leveraged clearly from the WiEye application's ongoing data collection.

\section{EVALUATION OF GSR}

In this section, we apply our GSR algorithm to analyze the factors that influence the performance of wireless networks and evaluate the gains of the WhiteCell structure.

\section{A. Experimental Setup}

We select a WhiteCell topology over a 7-unit cell with $1 \mathrm{~km}^{2}$ area, as depicted in Fig. 1. We use the in-field measurements from the previous section in GSR. For our analysis, all the cells are in considered to be the size of a single WiFi cell. The white space channels have more than 3 times the propagation range of WiFi channels even with the lowest frequency WiFi channel and the highest frequency white space channels according to the Friis transmission equation and the protocol model. Hence, one or more white space channels are assumed to be shared over the 7-unit cell.

The standby power consumption of a radio is set as 500 Watts and transmit power of 1 Watt per Mbps. The population density of the area is configured to 2000 per $\mathrm{km}^{2}$, the same as Dallas. The population distribution among the 7 cells are according to the measurement analysis shown in Fig. 12 at 11:00 AM. We set the traffic demand per user as 0.2 Mbps and assume $30 \%$ of the users will take the service (i.e., the take rate is $30 \%$ ). We assume the tolerated response time of users is $15 \mathrm{~ms}$ and adopt an 802.11n maximum data rate of $600 \mathrm{Mbps}$ for all radios. For the single white space channel setup, the first channel is assumed to be from the $800 \mathrm{MHz}$ band. For two white space channels, one channel is from the $450 \mathrm{MHz}$ band, the other is assumed to be from the $800 \mathrm{MHz}$ band. With three white space channels, we use two channels from the $800 \mathrm{MHz}$ band and one channel from the $450 \mathrm{MHz}$ band.

\section{B. Analysis in Extreme Scenarios}

First we investigate the impacts of channel quality and user mobility pattern on power consumption. In the measurements shown in Fig 3, the most available channel has a 1.5\% activity level, and the most busy channel has a $31.4 \%$ activity level. The area with the most user fluctuation has a $600 \%$ (Dallas C2) user variation, and the least fluctuation area has a $40 \%$ fluctuation. We have identified four scenarios based on these measurement extremes: A) low activity level and high user demand, B) high activity level and high user demand, C) low activity level and low user demand, and D) high activity level and low user demand.

We assume the user mobility varies linearly from the lowest amount of users to the highest amount of users. When the users are uniformly distributed in the cells, the power consumption will be the highest. The total users are represented as $100 \%$, each cell has $14.3 \%$. Thus, with $600 \%$ fluctuation, the user distribution noted in percentage varies from $2.3 \%$ to $14.3 \%$. To contrast the high fluctuation configuration, we set the low fluctuation scenario to vary form $6.9 \%$ to $9.7 \%$, maintaining the same average user distribution across time, $8.3 \%$, as the high fluctuation scenario. We assume that only a single white space channel is available in the simulation.

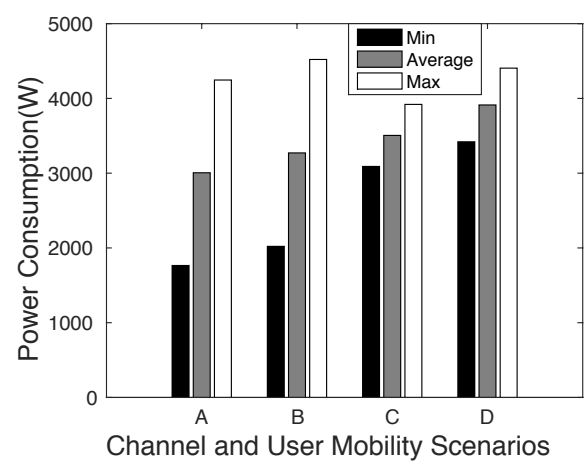

Fig. 8. Channel and User Mobility: A (Low Activity Level, High User Demand), B (High Activity Level, High User Demand), C (Low Activity Level, Low User Demand), and D (High Activity Level, Low User Demand)

From the simulation results shown in Fig. 8, the minimum power consumption in high user fluctuation (A and $\mathrm{B}$ ) is lower than in low user fluctuation (C and D) by $75.1 \%$ and $69.2 \%$, respectively. The average power consumption in high user fluctuation (A and B) is lower than the low user fluctuation (C and $\mathrm{D}$ ) by $16.3 \%$ and $18.6 \%$, respectively, but the peak power consumption is higher than in low user fluctuation by $7.7 \%$ and $2.6 \%$, respectively. When the number of users is lower, the white space radio will replace one or more $\mathrm{WiFi}$ or cellular radios to reduce the power consumption. Thus, the total and average power consumption is reduced by serving the traffic demand via less active radios. In contrast, with a higher activity level, the offloading system has to spend more power to achieve the same quality of service due to the reduction in capacity.

We also study the impact of differing response times, the user may tolerate $W$, on power consumption. The tolerance response time is varied from $5 \mathrm{~ms}$ to $90 \mathrm{~ms}$, using $5 \mathrm{~ms}$ steps.

From the results shown in Fig. 9, we see that the lower the requirement on response time, the less power is consumed. As the tolerance response time increases, the power consumption of both $\mathrm{WiFi}$ and heterogeneous configurations decrease 


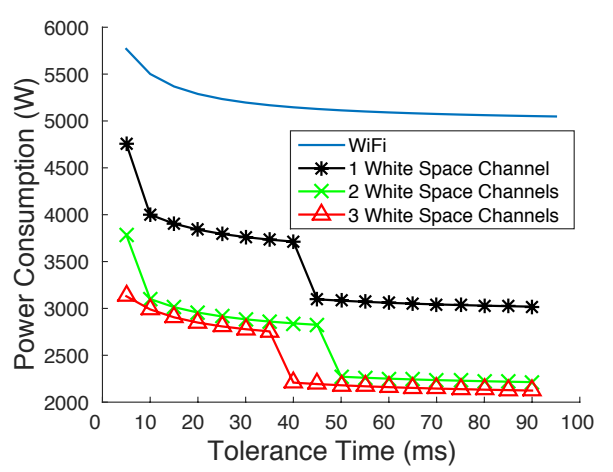

Fig. 9. Power Consumption across Delay Tolerance

monotonically. The WiFi configuration's power savings mainly stems from the reduction of channel capacity delivered. The white space configuration, however, saves power from both the reduction of channel capacity delivered as well as radios being disabled. With this in mind, we can see sharp reductions in power consumption using the white space configuration due to radios (seen in the step behavior of the curves). WiFi also experiences a reduction in power consumption, but it is more gradual. We observe the power consumption can be most greatly reduced by $151.2 \%$ with three white space channels under the tolerance response time of $90 \mathrm{~ms}$. When the tolerance response time is more than $50 \mathrm{~ms}$, there is a slight difference between the two white space channels and one white space channel. As discussed in the previous section, most of the major cities in the U.S. have restrictions on the number of white space channels available. Thus, the network carrier is able to estimate the quality of service they can offer according to the population density of the region, the number of white space channels available, the expected response time required, and the power available for operation.

Further, we utilize our previous measurement study of channel activity in multiple cities of the DFW metroplex to study the population density variation in white space network design [13]. For this analysis, we use the achieved channel capacity that maps to the population distribution.

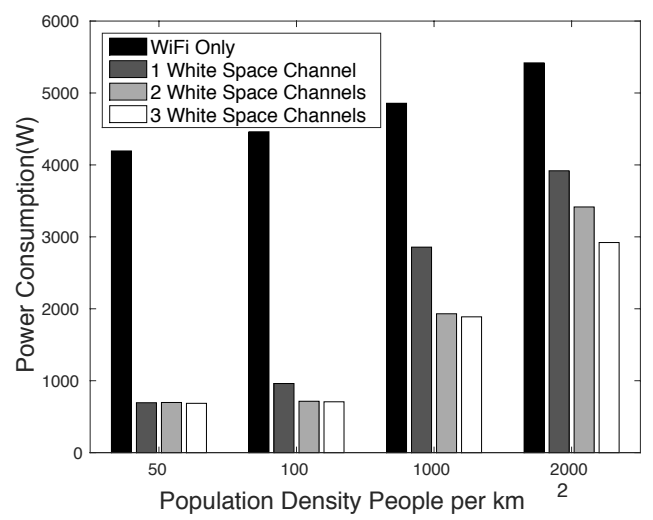

Fig. 10. Power Consumption across Population Distribution

From the results shown in Fig. 10, we see that as the population increases, all the network configurations require more power to serve the users. The power saving gains of a single white space channel reach their peak, $512.6 \%$, at a user density of 50 users $/ \mathrm{km}^{2}$, and the users are able to be satisfied by only

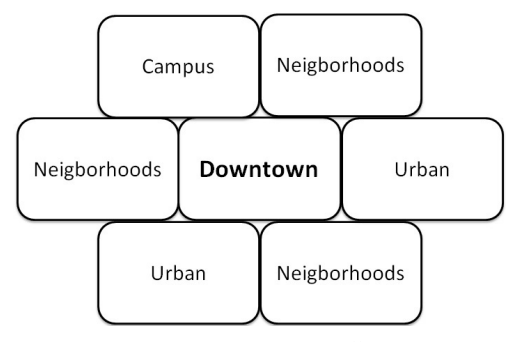

Fig. 11. Virtual City

one white space channel. The results provide a reference for carriers to choose the resource allocation method according to the population density. Increasing the number of white space channels does not provide any additional reduction in power consumption. We see a similar capping out at a user density of 100 users $/ \mathrm{km}^{2}$ with two white space channels available. The number of white space channels reaches a point of consistent power consumption increases with the population. At a user density of 50 users $/ \mathrm{km}^{2}$, one white space channel may be sufficient while 100 users $/ \mathrm{km}^{2}$ requires two white space channels. Also, the gain of a single white space channel decreases as the number of white space channels increases. This diminishing-returns effect on the gain can be seen at a user density of 1000 users $/ \mathrm{km}^{2}$ : the first white space channel gains $70 \%$ of power consumption, the second channel adds $48 \%$ to the system gains, and the third channel adds only $2.3 \%$. Therefore, when the number of white space channels are limited, splitting the channels to serve more WiFi cells could decrease power consumption, though the power saved decreases as the population density increases.

\section{Evaluation in Dallas-like Virtual City}

In practice, an area that has a stable channel state and constant user mobility does not exist. Thus, in-field measurements are required to infer the expected performance in the real world. To evaluate the system performance with a more practical configuration, we query multiple types of areas in Dallas to find the percentage of user activity across time. In a similar fashion to our prior in-field measurement database query, we pull measurements from downtown areas, urban areas, and suburban neighborhoods around Dallas in areas of fixed size equal to the area of SMU's campus.

Specifically, we use urban area measurements from the east of SMU's campus and from a shopping mall (North Park Mall). Suburban neighborhood area measurements were taken from the Highland Park residential area north of campus, from a residential area north of North Park Mall, and from The Village apartment complex east of campus. The number of users located in these area are counted according to the GPS locations. The distribution of the percentage of users across these areas during a given weekday is recorded in Fig. 12.

The measurements are put in a WhiteCell configuration as previously described, assuming the residents in the city are held constant. The channel variation across the spectrum is established according to both the channel state measurements as well as the user mobility from the measurement results of WiEye discussed in Section III. The results across 24 hours of the simulated city are shown in Fig. 13. 


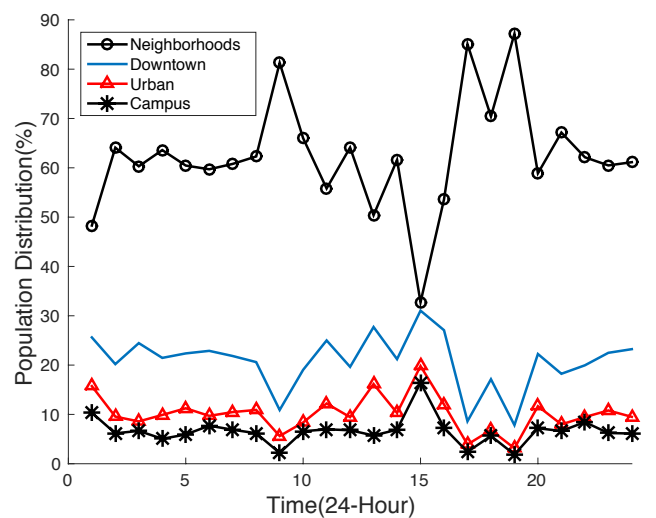

Fig. 12. User Distribution across Time

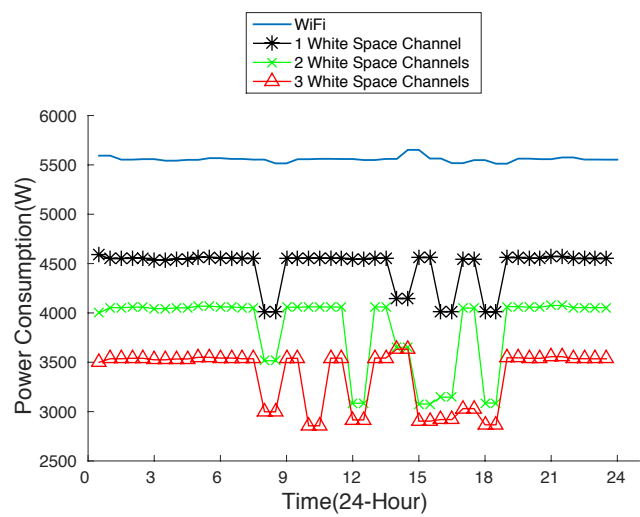

Fig. 13. Power Consumption across Time

In this simulation, we observe that the WiFi power consumption remains constant in cases when the users are more uniformly distributed. This is because the WiFi radios must be operating in order to serve the users due to the relatively-low propagation range. The white space radios have the advantage of adapting to the non-uniform user distribution and mobility. When the user distribution changes quickly (e.g., at 9:00 $\mathrm{AM})$, the white space configuration can reduce the power consumption by approximately $20 \%$ compared to the previous user distribution. Three white space channels reduce power consumption by nearly half. As mentioned earlier, the reduction in power consumption is mainly caused by the ability to disable the radios when the white space channels can be used instead of the WiFi channels. From the numerical simulation, one white space channel could reduce the power consumed by $23.5 \%$ on average over 24 hours. Additionally, our results show that using two white space channels reduces power consumption by $44.6 \%$, and using three white space channels reduces power consumption by $63.3 \%$. Similar to the previous result, as the number of white space channel increases, the power consumption gains per channel will become constant since there is enough channel capacity to satisfy the users. According to this result, we can design the offloaded network to utilize unlicensed bands more efficiently, complementing the existing cellular infrastructure.

\section{RELATED WORK}

Since FCC policy changes have made white space bands available for use in data networks, many works have stud- ied how to leverage white spaces [20]. In [3], the authors considered a cognitive method to avoid collision between white space data networks and TV broadcasting channels. Many other works increase the convenience of using white space databases (e.g., Microsoft's White Space Database [21]). Google has even visualized the licensed white space channels in U.S. cities with an API for both research and commercial use [22]. In our previous work, we studied point-to-point communication with white space bands [23], and wireless network deployment with many white space channels [13]. However, many of the major cities in the U.S. do not have many white space channels, which seems to advocate for these networks to supplement (and jointly used with WiFi) instead of supplant cellular networks in dense urban areas.

Multi-user diversity is a topic of focus in the late 1990s [24], where the channel quality varies across users due to differences in environmental factors. As a result, transmitted wireless signals are influenced by large-scale propagation, multi-path fading, and several other factors [25]. Previous work has also identified multi-user diversity for MIMO and beamforming applications [26], [27]. However, most of these works do not empirically derive in the field both the spectral activity and user mobility patterns that contribute greatly to multi-user diversity.

Applying white spaces to wireless networks is similar to the previous multi-channel works with the exception of greater propagation range. In [15], a multi-channel framework is formulated as a queuing system, and a Server-Side Greedy algorithm is proposed to optimize the throughput with low complexity. In [28], a Delay-based Queue-Side-Greedy algorithm is proposed with low complexity for optimal throughput and near-optimal delay. Lastly, a multi-objective optimization framework has been used to minimize energy consumption in a multi-channel multi-radio system [29]. Other works [30] focused on full-duplex technology. However, these works do not address minimizing the power consumption to achieve a certain quality of service and all assume an on-off channel model versus a measurement-driven residual capacity and user demand. Spectral diversity is isolated for a single user in [31]. In [32], multi-user dynamic channel access is proposed jointly considering the temporal and spectral diversity in a multichannel model. However, none of these works address the channel association problem in a multiple frequency band scenario such as is the case with $\mathrm{WiFi}$ and white spaces. White spaces have been applied in a scenario with spectral diversity in a previous work of ours [13]. Energy and spectral efficient study has been done for transmission delay [33], [34] and interference reducing [35]. However, these works fail to leverage white space frequencies for multi-user diversity in both spectral and temporal scenarios.

Previous works in real-time systems have focused on minimizing required hardware resources [36] or proven the capacity augmentation bounds for schedulers of parallel tasks [37] However, these works assume the parallel tasks have uniform servers. White space use was investigated in [14] via a queuing system, but only the homogeneous case was explored. In contrast, we study the performance of a heterogeneous queuing system with both white space channels and WiFi channels for 
cellular offloading.

\section{CONCLUSION}

In this paper, we considered the joint use of white space and WiFi channels for cellular offloading with a minimum power consumed and according to differing qualities of service. To achieve this goal, we performed spectral wardriving in a major metropolitan area and leveraged crowdsourced mobile phone data over multiple large metropolitan areas to infer user mobility and resulting network demand. Using these measurements, we proposed Greedy Server-side Replacement algorithm that was inspired by a heterogeneous queuing system. Further we investigated the performance in a virtual city to simulate the expected in-field performance. Through this simulation, we showed that using white space channels can greatly reduce the power consumption of such cellular offloading systems in most scenarios. From our results, we also saw the advantage of using white space channels in adapting user mobility to reduce power consumption. Through extensive analysis of spectrum utilization and user mobility, we showed that power consumption in WhiteCell offloaded networks can be reduced by up to $513 \%$. Finally, we considered the quality of service impact on power consumption and found that up to $151 \%$ of the power can be saved with only a slight relaxation of waiting times.

\section{ACKNOWLEDGMENTS}

We would like to thank the anonymous reviewers for their valuable feedback, which helped in improving the presentation of the paper. This work was in part supported by NSF grants: CNS-1150215 and CNS-1320442.

\section{REFERENCES}

[1] C. A. Balanis, Antenna theory: analysis and design. John Wiley \& Sons, 2012.

[2] Google, "Spectrum database," http://www.google.org/spectrum/whitespace/ 2013.

[3] P. Bahl, R. Chandra, T. Moscibroda, R. Murty, and M. Welsh, "White space networking with WiFi like connectivity," ACM SIGCOMM vol. 39, no. 4, pp. 27-38, 2009.

[4] Z. Lei and S. J. Shellhammer, "Ieee 802.22: The first cognitive radio wireless regional area network standard," IEEE communications magazine, vol. 47, no. 1, pp. 130-138, 2009.

[5] J. van de Beek, J. Riihijarvi, A. Achtzehn, and P. Mahonen, "Uhf white space in europe? quantitative study into the potential of the 470-790 mhz band," in New Frontiers in Dynamic Spectrum Access Networks (DySPAN), 2011 IEEE Symposium on. IEEE, 2011, pp. 1-9.

[6] S. Singh, H. S. Dhillon, and J. G. Andrews, "Offloading in heterogeneous networks: Modeling, analysis, and design insights," Wireles Communications, IEEE Transactions on, vol. 12, no. 5, pp. 2484-2497, 2013.

[7] A. Aijaz, H. Aghvami, and M. Amani, "A survey on mobile data offloading: technical and business perspectives," Wireless Communications IEEE, vol. 20, no. 2, pp. 104-112, 2013.

[8] B. Han, P. Hui, and A. Srinivasan, "Mobile data offloading in metropolitan area networks," ACM SIGMOBILE Mobile Computing and Communications Review, vol. 14, no. 4, pp. 28-30, 2011

[9] W. Vereecken, W. Van Heddeghem, M. Deruyck, B. Puype, B. Lannoo, W. Joseph, D. Colle, L. Martens, and P. Demeester, "Power consumption in telecommunication networks: overview and reduction strategies," Communications Magazine, IEEE, vol. 49, no. 6, pp. 62-69, 2011.

[10] J. B. Andersen, T. S. Rappaport, and S. Yoshida, "Propagation measurements and models for wireless communications channels," IEEE Communications Magazine, vol. 33, no. 1, pp. 42-49, 1995.

[11] H. T. Friis, "A note on a simple transmission formula," vol. 34, no. 5, pp. 254-256, May 1946.
[12] T. Rappaport, Wireless Communications, Principles \& Practice. Prentice Hall, 1996.

[13] P. Cui, H. Liu, D. Rajan, and J. Camp, "A measurement study of white spaces across diverse population densities," in in Proceedings of IEEE 2014 WiNMeE. IEEE, 2014, pp. 30-36.

[14] S. Chen, A. M. Wyglinski, S. Pagadarai, R. Vuyyuru, and O. Altintas, "Feasibility analysis of vehicular dynamic spectrum access via queueing theory model," Communications Magazine, IEEE, vol. 49, no. 11, pp. 156-163, 2011.

[15] S. Bodas, S. Shakkottai, L. Ying, and R. Srikant, "Low-complexity scheduling algorithms for multichannel downlink wireless networks,' Networking, IEEE/ACM Transactions on, vol. 20, 2012.

[16] S. Niida, S. Uemura, and H. Nakamura, "User tolerance for waiting time," IEEE Vehicular Technology Magazine, vol. 5, no. 3, pp. 61-67, 2010.

[17] P. Viswanath, D. N. C. Tse, and R. Laroia, "Opportunistic beamforming using dumb antennas," Information Theory, IEEE Transactions on, vol. 48, no. 6, pp. 1277-1294, 2002.

[18] S. Yu, R. Doss, T. Thapngam, and D. Qian, "A transformation model for heterogeneous servers," in High Performance Computing and Communications, 2008. HPCC'08. 10th IEEE International Conference on. IEEE, 2008, pp. 665-671.

[19] E. Gelenbe, G. Pujolle, and J. Nelson, Introduction to queueing net works. Citeseer, 1998.

[20] "Fcc white space," http://www.fcc.gov/topic/white-space, 2012

[21] "Microsoft research white space database", http://whitespaces.cloudapp.net/Default.aspx, 2013.

[22] "Google spectrum database," http://goo.gl/NnIFXQ, 2013.

[23] P. Cui, H. Liu, J. He, O. Altintas, R. Vuyyuru, D. Rajan, and J. Camp, "Leveraging diverse propagation and context for multi-modal vehicular applications," in IEEE WiVeC, 2013.

[24] R. W. Heath, M. Airy, and A. J. Paulraj, "Multiuser diversity for mimo wireless systems with linear receivers," in Signals, Systems and Computers, 2001. Conference Record of the Thirty-Fifth Asilomar Conference on, vol. 2. IEEE, 2001, pp. 1194-1199.

[25] Y. Gan and Y. Wu, "Multiple rayleigh fading channels modeling based on sum-of-sinusoids model," International Journal of Communication Systems, vol. 27, no. 11, pp. 2997-3012, 2014.

[26] Y. Du, E. Aryafar, P. Cui, J. Camp, and M. Chiang, "Samu: Design and implementation of selectivity-aware mu-mimo for wideband wifi," 2015.

[27] Y. Du, E. Aryafar, J. Camp, and M. Chiang, "ibeam: Intelligent clientside multi-user beamforming in wireless networks," in INFOCOM, 2014 Proceedings IEEE. IEEE, 2014, pp. 817-825.

[28] B. Ji, G. R. Gupta, X. Lin, and N. B. Shroff, "Performance of low-complexity greedy scheduling policies in multi-channel wireless networks: Optimal throughput and near-optimal delay," in INFOCOM 2013 Proceedings IEEE. IEEE, 2013, pp. 2589-2597.

[29] L. Liu, X. Cao, Y. Cheng, L. Du, W. Song, and Y. Wang, "Energyefficient capacity optimization in wireless networks," in INFOCOM, 2014 Proceedings IEEE. IEEE, 2014, pp. 1384-1392.

[30] Z. Tong and M. Haenggi, "Throughput analysis for wireless networks with full-duplex radios," in IEEE WCNC 2015, March 2015, pp. 717722 .

[31] T. Shu and M. Krunz, "Throughput-efficient sequential channel sensing and probing in cognitive radio networks under sensing errors," in Proceedings of the 15th annual international conference on Mobile computing and networking. ACM, 2009, pp. 37-48.

[32] Y. Liu and M. Liu, "To stay or to switch: Multiuser dynamic channel access," in IEEE INFOCOM 2013, 2013, pp. 1249-1257.

[33] G. Wang, J. Wu, and Y. R. Zheng, "Optimum energy and spectral efficient transmissions for delay-constrained hybrid arq systems," 2014

[34] J. Wu, G. Wang, and Y. R. Zheng, "Energy efficiency and spectral efficiency tradeoff in type-i arq systems," Selected Areas in Communications, IEEE Journal on, vol. 32, no. 2, pp. 356-366, 2014.

[35] Z. Tong and M. Haenggi, "Optimizing spatial reuse by dynamic power control," in IEEE ICC 2012, 2012, pp. 762-766.

[36] G. Nelissen, V. Berten, J. Goossens, and D. Milojevic, “Techniques optimizing the number of processors to schedule multi-threaded tasks,' in Real-Time Systems (ECRTS), 2012 24th Euromicro Conference on. IEEE, 2012, pp. 321-330.

[37] J. Li, J. J. Chen, K. Agrawal, C. Lu, C. Gill, and A. Saifullah, "Analysis of federated and global scheduling for parallel real-time tasks," in RealTime Systems (ECRTS), 2014 26th Euromicro Conference on. IEEE, 2014, pp. 85-96. 